\title{
ELECTRONICALLY TUNABLE LC HIGH PASS LADDER FILTER USING OTRA
}

\author{
Neeta Pandey, Venkatesh Kumar, Aayush Goel and Ankit Gupta \\ Department of Electronics and Communication Engineering, Delhi Technological University, India
}

\begin{abstract}
This paper presents Operational trans-resistance (OTRA) based electronically tunable high pass $L-C$ based ladder filter. The proposed high pass filter is designed by following a systematic approach using leap frog simulation for ladder filters. The cut-off frequency of the proposed filter can be varied with the application of appropriate voltages to the gate of MOSFETs hence making the design electronically tunable. Therefore, it can be used for RF applications at $455 \mathrm{kHz}$ and $1 \mathrm{MHz}$ (intermediate frequencies for $\mathrm{RF}$ applications). Performance of the proposed circuit is verified through SPICE simulations employing a $0.5 \mu \mathrm{m}$ MOSIS AGILENT CMOS realization of OTRA.
\end{abstract}

Keywords:

OTRA, High Pass Filter, Tunable

\section{INTRODUCTION}

The conventional voltage mode circuits and filters realized using operational amplifiers suffer from limitations which include lower bandwidth for higher gains. On the contrary, current-mode circuits offer several advantages as compared to voltage mode circuits such as higher bandwidth, increased linearity and reduced power consumption [1]-[4]. Continuous Time (CT) filters find extensive applications in fields like communication, measurements, and instrumentation and offer simplicity, low power and no sampling noise as compared to the discrete time filters. High pass filters form a key component in R/F microwave wireless applications [5]. Hence design of a current mode high pass filter has been proposed in this paper.

The main approach for designing of filters involves the following steps: (a) choice of an appropriate order according to the required design specifications, (b) the derivation of the corresponding transfer function, (c) synthesis and implementation of the required circuit from the obtained transfer function [6]. The synthesis of CT filters generally follows the approach of doubly terminated lossless LC ladder realization [7]-[8]. The resulting filter is tolerant to component variations, and offers an improved dynamic range performance and enhanced accuracy of passband magnitude response. Inductors are bulky and their use in integrated circuits is costly [7]. We can overcome this nonfeasibility of inductor realization in conventional LC ladder realization approach by using leap frog method, in which the relationship between various components is established through the signal flow graphs (SFG). Lossy and lossless active differentiators are then used to realize the obtained SFGs to implement the proposed design in this paper.

Literature survey of L-C ladder filters with different orders and approximation functions using leapfrog method [9]-[29] has been carried out. These configurations use variants of second generation current conveyors (CCII) namely dual/multiple output current controlled CCII (DOCCCII/ MOCCCII) [11]-[12], multiple output CCII (MOCCII) [10], differential voltage CC (DVCC) [14], differential voltage current controlled Current Feedback Operational amplifier (DVCCCFOA) [15] and differential voltage current controlled CCII (DVCCC), current feedback amplifier (CFA) [17], current feedback operational amplifier (CFOA) [16], current differencing buffer amplifier [18]-[20], current controlled current differencing buffer amplifier (CCCDBA) [21], operational trans-conductance amplifier [24]26], current backward trans-conductance amplifier [27], current differencing trans-conductance amplifier (CDTA) [22]-[23], CMOS based differential integrators [9], CMOS based lossy and lossless integrators [28]-[29].

From the available pool of literature, it can be summarized that, Configurations [15], [16], [21], [24]-[26] and [9]-[14], [17][20], [22], [23], [27]-[29] provide voltage and current outputs respectively. There is no appropriate impedance level for the configurations [13]-[15], [17]-[20], [24]-[26]. Thus an additional active block may be required to access the output. There is a limited literature is available on structures providing voltage output [15]-[17], [21], [24]-[26]. Furthermore, these structures do not provide output at low impedance except for those presented in [16], [21].

Table.1. Full form of some acronyms

\begin{tabular}{|c|c|}
\hline Acronym & Full form \\
\hline OTRA & Operational Trans-Resistance Amplifier \\
\hline SFG & Signal Flow Graphs \\
\hline CCII & Second generation Current Conveyors \\
\hline DVCC & Differential Voltage Current Conveyor \\
\hline DVCCFOA & $\begin{array}{c}\text { Differential Voltage Current Controlled Current } \\
\text { Feedback Operational Amplifier }\end{array}$ \\
\hline DVCCC & Differential voltage current controlled CCII \\
\hline CFA & Current Feedback Amplifier \\
\hline CFOA & Current Feedback Operational Amplifier \\
\hline CCCDBA & $\begin{array}{c}\text { Current Controlled Current Differencing Buffer } \\
\text { Amplifier }\end{array}$ \\
\hline CDTA & Current Differencing Trans-conductance \\
Amplifier
\end{tabular}

OTRA is a current mode active device with voltage as the output. It does not have any limitations on the slew rate and gain bandwidth product [30]-[35]. The parasitic effects are negligible as the internal terminals are grounded. Therefore OTRA can be easily and appropriately utilized for the design of filter with voltage output based on LC ladder network with Leapfrog simulation method.

The paper presented has been divided into five sections as described: Section 2 highlights leapfrog based simulation of LC- 
ladder, section 3 elaborates on the functioning of the proposed filter using OTRA block, section 4 describes the functioning of proposed filter considering non-idealities and section 5 presents simulation and results, which is finally followed by conclusion.

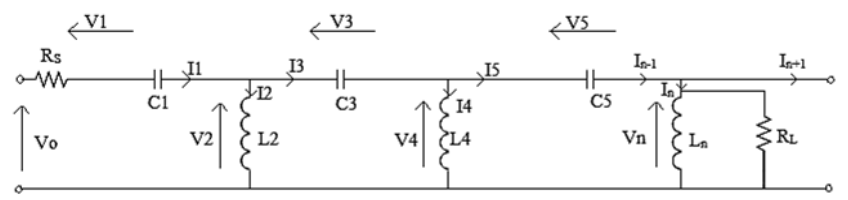

Fig. $1 . n^{\text {th }}$ order doubly terminated LC-Ladder

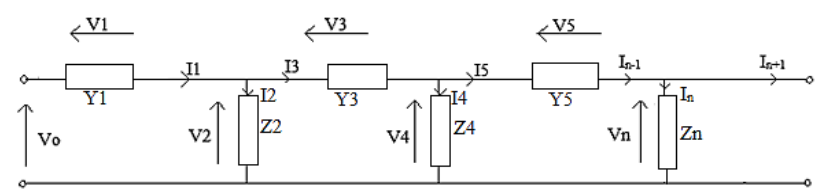

Fig. $2 . n^{\text {th }}$ order doubly terminated LC-Ladder with general immittances

\section{LC HP LADDER FILTER USING LEAP FROG SIMULATION}

This section illustrates and presents the realization of doubly terminated LC ladder with capacitors in series arm and inductors in the shunt arm to perform high pass operation. The currents and voltages in the shunt arm are given respectively as:

$$
I_{k}=I_{k-1}-I_{k+1}, \text { where }(k=2,4,6, \ldots, n-2)
$$

and

$$
V_{k}=V_{k-1}-V_{k+1}, \text { where }(k=1,2,3, \ldots, n-1)
$$

As the value of $I_{n}=0$ and $I_{n}=I_{n-1}$

$$
V_{n}=\frac{I_{k}=I_{k} s L_{k}}{\left[\frac{1}{s L_{n}}+G_{L}\right]} \text { with } G_{L}=1 / R_{L} .
$$

For the series arm, the currents are given as:

$$
\begin{gathered}
I_{k}=V_{k-1}-s C_{k+1}, \text { where }(k=4,6,8, \ldots, n) \\
I_{1}=\frac{V_{n}}{\left[\frac{1}{s L_{1}}+R_{s}\right]}
\end{gathered}
$$

The elements in the series arms are rewritten as Admittances whereas those in the shunt arm as impedances. The Fig.2 shows modification over Fig.1.

The node voltages and the current in series arm are related as:

$$
\begin{gathered}
I_{k}=Y_{k}\left(V_{k-1}-V_{k+1}\right), \text { where }(k=1,3,5, \ldots, n-1) \\
V_{k}=Z_{k}\left(I_{k-1}-I_{k+1}\right), \text { where }(k=2,4,6, \ldots, n-2) \\
V_{n}=Z_{n} I_{n-1}
\end{gathered}
$$

OTRA is used for the active realization of above equations. OTRA is a block which provides voltage output by processing input current difference. Therefore, the necessary changes for performing the voltage differencing operation are discussed in the following section.

\section{PROPOSED CIRCUIT}

The proposed sixth order high pass filter uses OTRA block which is in active building block. The first section briefly describes the properties and characteristics of OTRA block. The design of the proposed filter is described in the subsequent section.

\subsection{OTRA}

OTRA is an active three terminal device that consist of two input terminals and an output terminal as represented in Fig.3. It is a current mode device where the input terminals are virtually grounded leading to low input and output impedance. Thus, parasitic effects of OTRA can be neglected rendering it suitable for high frequency applications. The port relationship of OTRA is represented in Eq.(10).

$$
\left[\begin{array}{l}
V_{p} \\
V_{n} \\
V_{0}
\end{array}\right]=\left[\begin{array}{ccc}
0 & 0 & 0 \\
0 & 0 & 0 \\
R_{m} & -R_{m} & 0
\end{array}\right]\left[\begin{array}{c}
I_{p} \\
I_{n} \\
I_{0}
\end{array}\right]
$$

where, $R_{m}$ represents the trans-resistance gain of OTRA, which approaches infinity for ideal operation. Due to the high value of $R_{m}$, the two input currents are of same value. Therefore, OTRA is used in negative feedback configuration for linear circuit applications.

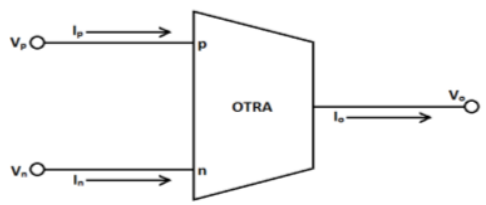

Fig.3. OTRA Circuit Symbol

\subsection{OTRA BASED IMPLEMENTATION OF PROPOSED LC HIGH PASSED LADDER FILTER}

Since OTRA provides current as output, for direct realization of Eq.(9) we multiply the equation by $R_{s c}$, the scaling resistance and Eq.(7) can be rewritten as:

$$
\begin{gathered}
R_{S C} I_{k}=R_{S C} Y_{k}\left(V_{k-1}-V_{k+1}\right), \text { where }(k=1,3,5, \ldots, n-1) \\
V_{I k}=t_{Y K}\left[V_{k-1}-V_{k+1}\right], \text { where }(k=1,3,5, \ldots, n-1)
\end{gathered}
$$

where,

$$
\begin{gathered}
V_{I k}=R_{S C} I_{k} \\
Y_{1}=\frac{1}{\left[\frac{1}{s C_{1}}+R_{s}\right]}, \\
Y_{k}=s C_{k} \text { and } t_{Y k}=R_{S C} Y_{k} \quad(k=1,3,5, \ldots, n-1)
\end{gathered}
$$

The node voltages in Eq.(8) and Eq.(9) are rewritten as Eq.(13), Eq.(14) and Eq.(15) with the help of Eq.(11) and Eq.(12).

$$
\begin{gathered}
V_{k}=\frac{Z_{k}}{R_{S C}}\left(R_{S C} I_{k-1}-R_{S C} I_{k+1}\right)(k=2,4,6, \ldots, n-2) \\
V_{k}=t_{Z k}\left[V_{I(k-1)}-V_{I(k+1)}\right](k=2,4,6, \ldots, n-2) \\
V_{n}=t_{Z n} V_{I(n-1)}
\end{gathered}
$$

where, 


$$
\begin{gathered}
Z_{k}=s L_{\mathrm{k}}, \\
Z_{n}=\frac{1}{\left[\frac{1}{s L_{n}}+G_{L}\right]}
\end{gathered}
$$

and

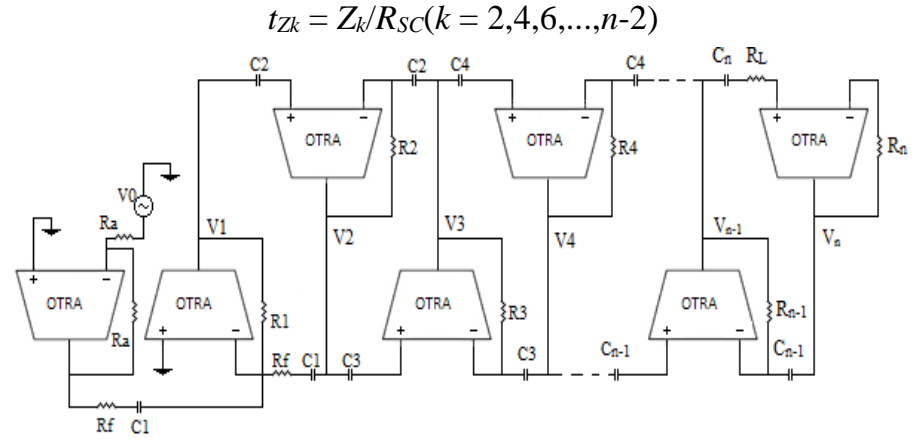

Fig.5. Proposed OTRA based $n^{\text {th }}$ order doubly terminated HPF

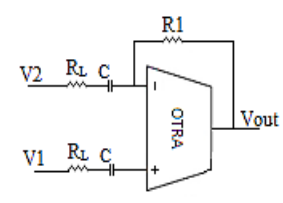

Fig.4. Non-Ideal Differentiator

OTRA block can be used to implement an ideal (lossless) and a non-ideal (lossy) differentiator. Using these two differentiators $n^{\text {th }}$ order high pass filter has been proposed based on the leapfrog simulation.

The Eq.(11) to Eq.(15) are actively realized differencing lossy and lossless differentiators. A lossy differentiator as the first stage is followed by $(n-2)$ lossless differentiator stages. The $n^{\text {th }}$ stage, i.e. the output stage is again a lossy differentiator.

The circuit for the non-ideal differentiator is represented in Fig.4. The output voltage in terms of $V_{1}$ and $V_{2}$ is given as:

$$
V_{\text {out }}=R_{1}\left[V_{1}\left(s C+G_{L}\right)-V_{2}\left(s C+G_{L}\right)\right]
$$

For the first stage the non-ideal circuit is designed using an inverter stage followed by a summer stage as shown in Fig.5.

\subsection{ELECTRONIC TUNABILITY}

More attention is being given to electronically tunable components as traditional electronic components may have deviations in fine-tuning the tolerances of the electronic components [36]. Electronic tunability enhances the controllability of a microcomputer or microcontroller [37]. A tunable high pass OTRA filter could be simulated by using NMOS instead of the feedback resistances so as to change the value of resistances w.r.t. the input voltages at the gate of NMOS and hence change the cut-off frequency of the proposed filter. The Fig.6 represents a single stage tunable lossy differentiator with the value of resistance given by Eq.(17).

$$
R_{i}=\frac{1}{\mu_{n} C_{o x}\left(\frac{W}{L}\right)\left(V_{a i}-V_{b i}\right)}
$$

Thus, electronic tunability can be embedded by implementing resistors $R_{1}$ to $R_{n}$ with the help of MOS based realization using method. This makes the design flexible and the filter can be finetuned in case there is any deviation from desired response. The modified Fig.7 shows tunable filter implementations where the gate voltages $V_{a i}$ and $V_{b i}$ realize the required resistors using MOS. $(i=1,2,3, \ldots, n)$.

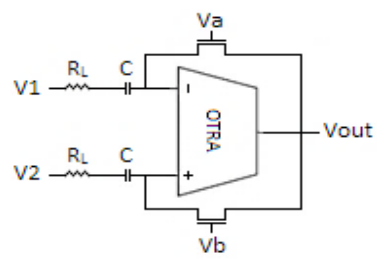

Fig.6. Single stage Tunable Differentiator

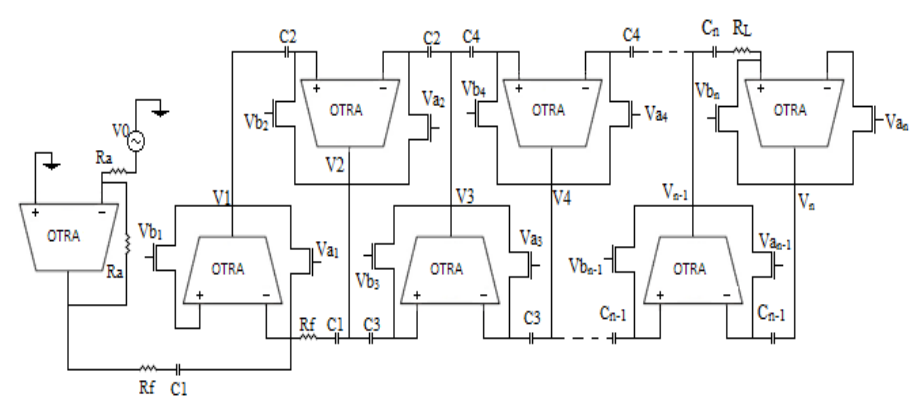

Fig.7. Electronically tunable Proposed OTRA based $n^{\text {th }}$ order doubly terminated HPF

\subsection{NON-IDEALITY ANALYSIS}

The trans-resistance gain $\left(g_{m}\right)$ of an ideal OTRA block is infinity causing the input currents to become equal. But in reality the gain is finite for small bandwidth and decreases further with increase in frequency. Therefore effects of this non-ideality should be included while deriving transfer functions.

The single pole model of the OTRA block will have transresistance gain of:

$$
R_{m}(s)=R_{0} /\left(1+\left(s / \omega_{0}\right)\right)
$$

At high frequencies the Eq.(18) can be approximated to:

$$
R_{m}(s)=1 / s C_{P}
$$

where, the parasitic capacitance $C_{p}=1 / R_{0} \omega_{0}$, open loop DC transresistance gain is $R_{0}$ and trans-resistance cut-off frequency is $\omega_{0}$.

The transfer function of single stage differentiator is modified to

$$
V_{\text {out }}=R_{1}\left\{V_{1}\left[s\left(C+C_{p}\right)+G_{L}\right]-V_{2}\left[s\left(C+C_{p}\right)+G_{L}\right]\right\}
$$

\section{SIMULATIONS AND RESULTS}

The proposed high-pass filter is realised using lossy and lossless differentiator implementations of OTRA on the lines of a sixth-order Chebyshev filter with a $0.1 \mathrm{~dB}$ ripple width in passband. The passive elements used in the prototype Chebyshev filter has normalized values of $C_{1}=0.8561, L_{2}=0.7122, C_{3}=$ $0.4863, L_{4}=0.6592, C_{5}=0.5255, L_{6}=1.1604$ and $R_{F}=0.7378$. For a cut-off frequency of $455 \mathrm{kHz}, R_{a}=1 \mathrm{k} \Omega, R_{f}=0.599 \mathrm{k} \Omega$ and $C_{a}=C_{b}=C_{c}=C_{d}=C_{e}=C_{f}=0.5 \mathrm{nF}$ the de-normalized values of 
the feedback path resistors are $R_{1}=0.99 \mathrm{k} \Omega, R_{2}=0.299 \mathrm{k} \Omega, R_{3}=$ $0.567 \mathrm{k} \Omega, R_{4}=0.277 \mathrm{k} \Omega, R_{5}=0.613 \mathrm{k} \Omega, R_{6}=0.487 \mathrm{k} \Omega$ and $R_{L}=$ $1.1 \mathrm{k} \Omega$.

Table.2. Aspect ratios of transistors in Fig. 8

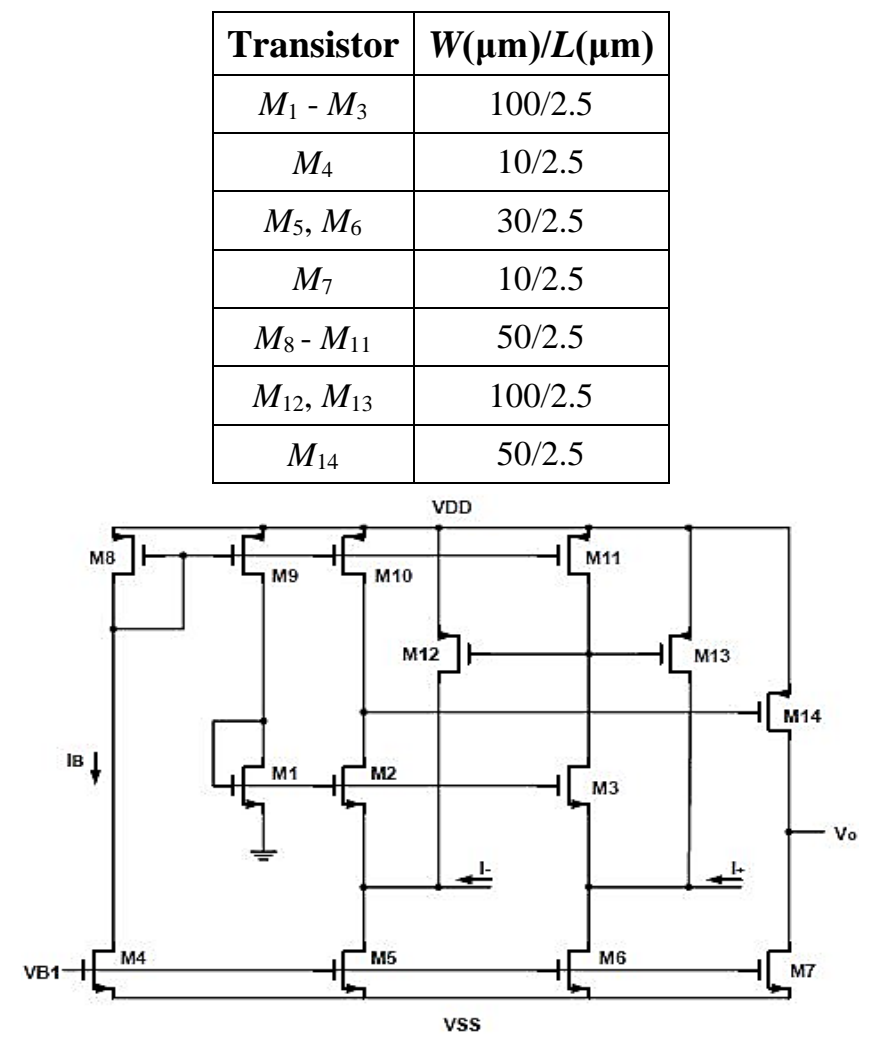

Fig.8. CMOS Implementation of OTRA [38]

The simulated frequency response of a high-pass LC-ladder filter is shown in Fig.9. Also the frequency response of OTRA based ladder filter in superimposed to know the deviations of the active realization. The simulated frequency response of a tunable OTRA based filter for cut-off frequencies of $455 \mathrm{kHz}$ and $1 \mathrm{MHz}$ are shown in Fig.10. Using Eq.(17) the aspect ratios (W/L) of the MOS transistors used to attain tunability are calculated as shown in Table.3. For achieving the cut-off frequency of $455 \mathrm{kHz}$ we have set the gate voltages of MOS resistors in feedback path to $V a_{i}=0.9 \mathrm{~V}$ and $V b_{i}=1.2 \mathrm{~V}$, while to achieve the cut-off frequency of $1 \mathrm{MHz}$ we have to set the gate voltages, $V a_{i}$ and $V b_{i}$ to $0.75 \mathrm{~V}$ and $1.4 \mathrm{~V}$ respectively $(i=1,2,3 \ldots \ldots 6)$.

Table.3. Aspect Ratios of Transistors in Fig.8

\begin{tabular}{|c|c|}
\hline Transistor & $W(\mu \mathrm{m}) / L(\mu \mathrm{m})$ \\
\hline$M a_{1}-M b_{1}$ & $2.7 / 2.5$ \\
\hline$M a_{2}-M b_{2}$ & $4.33 / 5$ \\
\hline$M a_{3}-M b_{3}$ & $5 / 2.73$ \\
\hline$M a_{4}-M b_{4}$ & $4.75 / 5$ \\
\hline$M a_{5}-M b_{5}$ & $5 / 2.96$ \\
\hline$M a_{6}-M b_{6}$ & $2.68 / 5$ \\
\hline
\end{tabular}

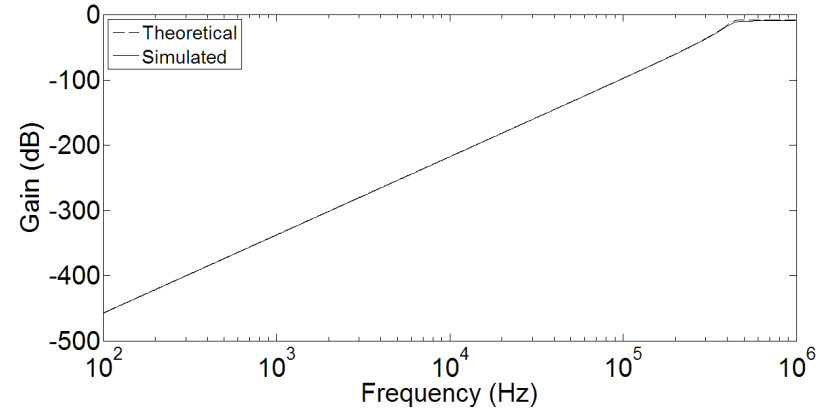

Fig.9. Simulated OTRA based vs Theoretical LC-ladder Filter Frequency Response

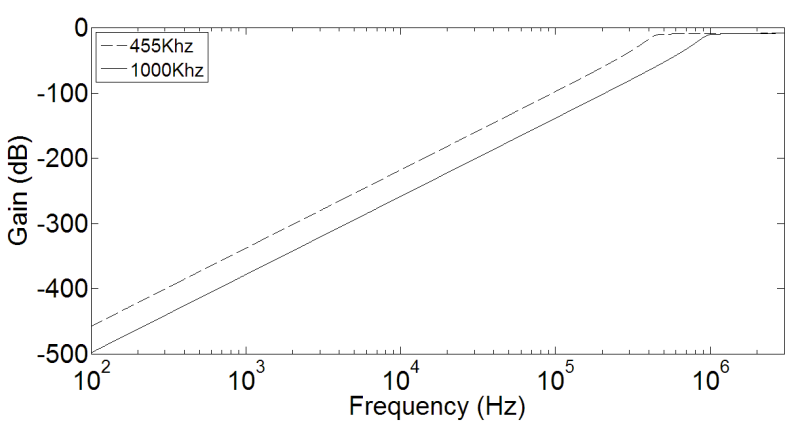

Fig.10. Simulated OTRA based Tunable Filter Frequency Response for $455 \mathrm{kHz}$ and $1 \mathrm{MHz}$

\section{CONCLUSION}

OTRA based sixth order high-pass filter is presented and simulated in this paper. As compared to Current feedback operational amplifier (CFOA) based high pass ladder filter presented in [39], the proposed OTRA based sixth order high pass ladder filter is electronically tunable. The proposed topology also offers an advantage in terms of higher operating and cut-off frequencies as compared to existing high-pass ladder filter designs [39]. Simulation and results are presented for $455 \mathrm{kHz}$ and 1Mhz. Non-Ideality Analysis has been included along with electronic tunability specifications.

\section{REFERENCES}

[1] H.P. Chen and P.L. Chu, "Versatile Universal Electronically Tunable Current-Mode Filter using CCIIs", IEICE Electronics Express, Vol. 6, No. 2, pp. 122-128, 2009.

[2] B. Wilson, "Recent Developments in Current Conveyor and Current-Mode Circuits", IEE Proceedings-Circuits, Devices and Systems, Vol. 137, No. 2, pp. 63-77, 1990.

[3] G.W. Roberts and A.S. Sedra, "All Current-Mode Frequency Selective Circuits", Electronics Letters, Vol. 25, No. 12, pp. 759-761, 1989.

[4] C.M. Chang, "Current Mode Allpass/Notch and Bandpass Filter using Single CCII", Electronics Letters, Vol. 27, No. 20, pp. 1812-1813, 1991.

[5] Hui Chen, Di Jiang, Ke-Song Chen and Hong-Fei Zhao, “A Compact High-Pass Filter using Hybrid Microstrip/Nonuniform CPW with Dual-Mode Resonant Response", International Journal of Antennas and Propagation, Vol. 2016, pp. 1-7, 2016. 
[6] Suman Kumari, Stuti Gupta, Neeta Pandey, Rajeshwari Pandey and Rashika Anurag, "LC-Ladder Filter Systematic Implementation by OTRA", International Journal Engineering Science and Technology, Vol. 19, No. 4, pp. 1808-1814, 2016.

[7] A. Thanachayanont and A. Payne, "CMOS Floating Active Inductor and its Application to Bandpass Filter and Oscillator Designs", IEE Proceedings-Circuits, Devices and Systems, Vol. 147, No. 1, pp. 42-48, 2000.

[8] R. Shaumann and M.E. Van Valkenburg, "Design of Analog Filters", Oxford University Press, 2001.

[9] S. Sa-Ad and D. Chaythong, "A High Frequency Current Mode Ladder Filter using Multiple Output Lossless Integrator", Proceedings of International Symposium in Intelligent Signal Processing and Communication systems, pp. 1-4, 2008.

[10] J. Wu and E. El-Masry, "Current-Mode Ladder Filters using Multiple Output Current Conveyors", IEE ProceedingsCircuits, Devices and Systems, Vol. 143, No. 4, pp. 218-222, 1996.

[11] A. Jiraseree-Amornkunt, N. Fujii and W. Surakampontorn, "Realisation of Electronically Tunable Ladder Filters using Multi-Output Current Controlled Conveyors", Proceedings of IEEE International Symposium on Circuits and Systems, pp. 541-544, 2003.

[12] A. Campeanu and J. Gal, "Systematic Implementation Method of LC-Ladder Filter by MO-CCCII Circuits", Proceedings of $5^{\text {th }}$ International Congress and Exhibition of Electrical and Electronic Engineering, pp. 1-5, 2007.

[13] X. Yanhui and H. Peng, "Realisation of Low Pass and BandPass Leapfrog Filters using OAs and CCCIIs", Proceedings of IEEE International Conference Management and Service Science, pp. 1-4, 2009.

[14] Y.H. Xi and X. Li, "Active Simulation of passive Leapfrog Ladder Filters Using DVCCs", Proceedings of IEEE International Conference on Industrial Technology, pp. 1-5, 2008.

[15] C. Laoudias and C. Psychalinos, "Comparative Study of Resistor Less Filters using Differential Voltage Current Controlled Current Feedback Operational Amplifiers and Differential Voltage Current Controlled Current Conveyors", International Scholarly Research Notices, Vol. 2013, pp. 7-11, 2013.

[16] P.K. Sinha, A. Saini, P. Kumar and S. Mishra, "CFOA based Low Pass and High Pass Ladder Filter-a New Configuration", Circuits Systems, Vol. 5, No. 12, pp. 293300, 2014.

[17] T.S. Rathore and U.P. Khot, "CFA-based GroundedCapacitor Operational Simulation of Ladder Filters", International Journal of Circuit Theory and Applications, Vol. 36, No. 5-6, pp. 697-716, 2008.

[18] W. Tangsrirat, N. Fujii and W. Surakampontorn, "CurrentMode Leapfrog Ladder Filters using CDBAs", Proceedings of IEEE International Symposium on Circuits and Systems, pp. 57-60, 2002.

[19] W. Tangsrirat, W. Surakampontorn and N. Fujii, "Realization of Leapfrog Filters using Current Differential Buffered Amplifiers", The Institute of Electronics, Information and Communication Engineers, Vol. 86, No. 2, pp. 318-326, 2003.
[20] W. Tangsrirat, K. Klahan, T. Dumawipata and W. Surakampontorn, "Low-Voltage NMOS-based Current Differencing Buffered Amplifier and its Application to Current-Mode Ladder Filter Design", International Journal of Electronics, Vol. 93, No. 11, pp. 777-791, 2006.

[21] V. Ramola, S. Mishra, R.K. Singh and D.S. Chauhan, "CCCDBA based Implementation of Voltage Mode Third Order Filter", Proceedings of International Conference on Advances in Communication, Network, and Computing, pp. 185-192, 2012.

[22] A. Uygur and H. Kuntman, "Seventh-Order Elliptic Video Filter with $0.1 \mathrm{~dB}$ Pass Band Ripple Employing CMOS CDTAs", AEU-International Journal of Electronics and Communications, Vol. 61, No. 5, pp. 320-328, 2007.

[23] J. $\mathrm{Xu}$ and $\mathrm{C}$. Wang, "The Current Difference Transconductance Amplifier (CDTA)", Available at: https://www.edn.com/design/analog/4405810/The-CurrentDifferencing-Transconductance-Amplifier-CDTA.

[24] Y. Sun, "Synthesis of leap-frog multiple-loop feedback OTA-C filters", IEEE Transactions on Circuits and Systems II: Express Briefs, Vol. 53, No. 9, pp. 961-965, 2006.

[25] H.W. Su and Y. Sun, "A CMOS $100 \mathrm{MHz}$ Continuous-Time Seventh Order 0.05 Equiripple Linear Phase Leapfrog Multiple Loop Feedback GM-C Filter", Proceedings of IEEE International Symposium on Circuits and Systems, pp. 17-20, 2002.

[26] C. Feng, "The Design of OTA-C Filter based on the Prototype of Ladder LC-Filter", Journal of Theoretical and Applied Information Technology, Vol. 49, No. 1, pp. 144148, 2013.

[27] U.E. Ayten, M. Sagba and H. Sedef, "Current Mode Leap Frog Ladder Filters using a New Active Block", AEUInternational Journal of Electronics and Communications, Vol. 64, No. 6, pp. 503-511, 2010.

[28] P. Prommee and N. Wongprommoon, "Tunable CMOSbased Current Mode Fifth Order Ladder Low-Pass Filter", Proceedings of $36^{\text {th }}$ IEEE International Conference on Telecommunications and Signal Processing, pp. 397-401, 2013.

[29] T. Kunto, P. Prommee and M.T. Abuelmaatti, "Electronically Tunable Current-Mode High-Order Ladder Low-Pass Filters based on CMOS Technology", Radioengineering, Vol. 24, No. 4, pp. 974-987, 2015.

[30] K.N. Salama and A.M. Soliman, "CMOS Operational Transresistance Amplifier for Analog Signal Processing”, Microelectronics Journal, Vol. 30, No. 3, pp. 235-245, 1999.

[31] R. Pandey, N. Pandey, M. Bothra and S.K. Paul, "Operational Transresistance Amplifier based Multiphase Sinusoidal Oscillator", Journal of Electrical and Computer Engineering, Vol. 2011, pp. 1-8, 2011.

[32] C.L. Hou, H.C. Chien and Y.K. Lo, "Square Wave Generators Employing OTRAs", IEE Proceedings-Circuits, Devices and Systems, Vol. 152, No. 6, pp. 718-722, 2005.

[33] Y.K. Lo, H.C. Chien and H.G. Chiu, "Switch Controllable OTRA based Bistable Multivibrators", IET Circuits, Devices and Systems, Vol. 2, No. 4, pp. 373-382, 2008.

[34] A. Gupta, R. Senani, D.R. Bhaskar and A.K. Singh, "OTRAbased Grounded-FDNR and Grounded-Inductance Simulators and their Applications", Circuits, Systems, and Signal Processing, Vol. 31, No. 2, pp. 489-499, 2012. 
[35] A. Gokcen, S. Kilinc and U. Cam, "Fully Integrated Universal Biquads using Operational Transresistance Amplifier with MOS-C Realisation", Turkish Journal of Electrical Engineering and Computer Sciences, Vol. 19, No. 3, pp. 363-372, 2011.

[36] H.P. Chen, Y.S. Hwang and Y.T. Ku, "Voltage-Mode and Current-Mode Resistorless Third-Order Quadrature Oscillator", Applied Sciences, Vol. 7, No. 2, pp. 179-189, 2017.

[37] Surasak Sangyaem, Surapong Siripongdee, Winai Jaikla and Fabian Khateb, "Five-Inputs Single-Output Voltage Mode
Universal Filter with High Input and Low Output Impedance using VDDDAs", Optik-International Journal for Light and Electron Optics, Vol. 128, pp. 14-25, 2017.

[38] H. Mostafa and A.M Soliman, "A Modified CMOS Realization of the Operational Transresistance Amplifier (OTRA)", Frequenz, Vol. 60, No. 3-4, 2006.

[39] P. Sinha, A. Saini, P. Kumar and S. Mishra, "CFOA based Low Pass and High Pass Ladder Filter-A New Configuration", Circuits and Systems, Vol. 5, No. 12, pp. 293-300, 2014. 